Originalien

Zbl Arbeitsmed 2021 $\cdot 71: 8-18$ https://doi.org/10.1007/s40664-020-00409-3 Eingegangen: 7. Mai 2020

Überarbeitet: 31. Juli 2020

Angenommen: 15. September 2020

Online publiziert: 12 . Oktober 2020

(c) Der/die Autor(en) 2020

Norbert Kersten · Maren Formazin · Grit Müller

Fachbereich Arbeit und Gesundheit, Bundesanstalt für Arbeitsschutz und Arbeitsmedizin, Berlin, Deutschland

\title{
Burnout und psychosoziale Arbeitsbedingungen in Berufsgruppen mit hohen kognitiven Anforderungen
}

lisierung auf Beschäftigte, die im Bereich der menschenbezogenen Dienstleistungen tätig sind, bspw. Pflegekräfte und Lehrkräfte.

Spätere Arbeiten zeigten, dass Burnout nicht auf bestimmte Berufsgruppen beschränkt ist, sondern in nahezu allen Berufsgruppen beobachtet werden kann $[6,9,11]$. Bakker et al. betrachten Burnout als ein arbeitsbezogenes StressSyndrom, welches zwei Kerndimensionen - Erschöpfung und Sich-Distanzieren (Disengagement) - umfasst $[6,11]$, wobei Erschöpfung als Konsequenz intensiver physischer, affektiver und kognitiver Beanspruchung definiert wird, d.h. als eine langfristige Konsequenz aus der Exposition gegenüber spezifischen Arbeitsanforderungen [10].

Die Relevanz von Burnout leitet sich aus seinen Konsequenzen ab, die sowohl für Betroffene selbst als auch die Betriebe und die Gesellschaft als Ganzes bestehen, wie bereits Maslach und Kollegen in den 1990er-Jahren zusammenfassend feststellten [17]. So erhöhte das Vorliegen einer Burnout-Symptomatik zu Studienbeginn in einer finnischen Bevölkerungsstudie die Wahrscheinlichkeit, vier Jahre später eine Erwerbsminderungsrente zu beziehen, wobei dieser Effekt vor allem bei einer schweren BurnoutSymptomatik auftrat und auch nach Berücksichtigung weiterer soziodemographischer und gesundheitlicher Indikatoren statistisch signifikant blieb [1]. Weiterhin wies die Hälfte derjenigen, die eine schwere Form von Burnout hatten, zugleich eine depressive Störung auf, vor- rangig eine „major depression“ [3]. In einer weiteren Studie waren erhöhte Burnout-Werte mit einem Anstieg des Risikos für die Einnahme von Psychopharmaka (bspw. Antidepressiva) assoziiert [16].

Dass Burnout auch im Kontext von sicherheitsrelevantem Verhalten zu berücksichtigen ist, verdeutlicht eine Längsschnittstudie von Beschäftigten der finnischen Forstwirtschaft: Erhöhte Burnout-Werte zu Studienbeginn waren bis zu acht Jahre später mit einem erhöhten Risiko für schwere Verletzungen assoziiert, insbesondere bei stärker ausgeprägten Burnout-Werten [4].

Gemäß des Job-Demand-ResourcesModells, welches Arbeitsbedingungen in die zwei breiten Kategorien Anforderungen (,job demands“) und Ressourcen (,job ressources") kategorisiert, die differenziell mit verschiedenen Kriterienmaßen assoziiert sind [9], sollten hohe Anforderungen, die die Energiereserven der Beschäftigten aufbrauchen, $\mathrm{zu}$ Erschöpfung führen [6, 9]. Geringe Ressourcen sollten zu einem Rückzug von der Arbeit und in der Folge zum „disengagement“ führen [9], wobei sich empirisch zeigte, dass auch geringe Ressourcen mit hohen Erschöpfungswerten assoziiert sind [6].

Im deutschsprachigen Raum wurden hohe quantitative Anforderungen und negative soziale Beziehungen als Prädiktoren für Burnout identifiziert, während bei hoher Unterstützung durch Kollegen und Vorgesetzte die Burnout-Werte geringer waren; allerdings handelt es sich 
dabei nur um eine querschnittliche Erhebung [22].

Im Längsschnitt waren in einer Stichprobe von Beschäftigten der menschbezogenen Dienstleistungen hohe quantitative und emotionale Arbeitsanforderungen, hohes Arbeitstempo, Rollenkonflikt, geringe Vorhersagbarkeit sowie geringe Entwicklungsmöglichkeiten mit arbeitsbezogenem Burnout assoziiert; allerdings verblieb bei zusätzlicher Berücksichtigung des Burnout-Werts zu Studienbeginn nur geringe Vorhersagbarkeit als signifikanter Prädiktor [8].

In einem systematischen Review kommen Seidler et al. zu dem Schluss, dass ungünstige Arbeitsbedingungen - insbesondere hohe Anforderungen, aber auch geringe soziale Unterstützung - mit Burnout, v.a. seiner Kernkomponente, der emotionalen Erschöpfung, assoziiert sind [28]. Ein aktuelleres systematisches Review zeigt, dass soziale Unterstützung, Handlungsspielraum, Gerechtigkeit und Belohnung protektive Faktoren gegenüber Erschöpfung sind, während hohe Anforderungen, eine hohe Arbeitsmenge und Arbeitsplatzunsicherheit das Risiko für Erschöpfung erhöhen [5]. Diese Befunde unterstützen die Aussage von Ahola und Kollegen, Burnout als ein ernstzunehmendes Signal ungünstiger Arbeitsbedingungen zu betrachten [2].

Hohe kognitive Anforderungen bei der Arbeit bedeuten, dass Beschäftigte viele Aspekte gleichzeitig im Blick behalten und/oder sich merken müssen, neue Ideen entwickeln und weitreichende Entscheidungen treffen müssen [21]. In Berufsgruppen, die sich durch derartige hohe kognitive Anforderungen auszeichnen wie Publizist/innen, Werbefachkräfte und Softwareentwickler/ innen $[18,26]$, sind erhöhte BurnoutWerte mit einem Anstieg des Risikos für die Einnahme verschreibungspflichtiger Medikamente oder illegaler Substanzen zur Verbesserung der Leistungsfähigkeit oder des psychischen Wohlbefindens assoziiert [18].

Nicht betrachtet wurde in diesem Zusammenhang bisher, ob diese Berufsgruppen insgesamt erhöhte BurnoutWerte gegenüber der Beschäftigtenpopulation aufweisen - wobei hierzu eine repräsentative Vergleichsstichprobe er- forderlich ist - und ob Arbeits- und Beschäftigungsbedingungen bei ihnen in ähnlicher Weise mit Burnout assoziiert sind wie in der Beschäftigtenpopulation [18]. Das Ziel der vorliegenden Studie ist es, Burnout und seine Antezendenzen in diesen drei Berufsgruppen $\mathrm{zu}$ untersuchen.

Als Vergleichsstichprobe werden Personen aus einer repräsentativen Studie der Beschäftigtenpopulation Deutschlands herangezogen [24]. Das soll Einblick darüber geben, ob das BurnoutNiveau in den drei oben genannten Berufsgruppen höher ist als in der Beschäftigtenpopulation. Darüber hinaus wird der Zusammenhang zwischen psychosozialen Arbeitsbedingungen und Burnout untersucht sowie geprüft, ob dieser sich zwischen den drei Berufsgruppen und der Beschäftigtenpopulation unterscheidet.

\section{Studiendesign und Unter- suchungsmethoden}

\section{Stichproben}

Die Daten stammen aus zwei Querschnittsstudien: (1) der Studie „Einfluss psychischer Belastungen am Arbeitsplatz auf das Neuroenhancement " und (2) der ersten Welle der „Studie zur Mentalen Gesundheit bei der Arbeit“ (S-MGA) der Bundesanstalt für Arbeitsschutz und Arbeitsmedizin. Die Datenbasis für die Stichprobenziehungen bilden die Integrierten Erwerbsbiografien (IEB) der sozialversicherungspflichtig Beschäftigten, die aus den Meldungen der Bundesagentur für Arbeit (BA) beim Institut für Arbeitsmarkt- und Berufsforschung (IAB) zusammengeführt werden.

In der Neuroenhancement-Studie wurden Daten zu vier Berufsgruppen in Deutschland erhoben: angestellte Krankenhausärzt/innen, Publizist/ innen, Werbefachleute und Softwareentwickler/innen; die Ärzt/innen wurden in einer gesonderten Studie analysiert [23]. Es wurde ein zweistufiges Cluster-Stichprobenverfahren angewendet: erstens eine Gemeindestichprobe mit 175 Sample Points und zweitens eine Repräsentativstichprobe für jeden der vier Berufe proportional verteilt auf die Regional- klumpen (Stichtag: 31.12.2010). Die Auswahl der Gemeinden wurde auf solche mit mindestens 50.000 Einwohnern beschränkt, um für jede Berufsgruppe einen angemessenen Stichprobenumfang $\mathrm{zu}$ gewährleisten. Werbefachleute und Publizist/innen wurden über die Berufskennziffer (BKZ) im Beschäftigtenregister identifiziert. Eine direkte Identifizierung von Softwareentwickler/ innen war nicht möglich. Daher erfolgte die Stichprobenziehung aus der Gruppe der Datenverarbeiter/innen, die dann in einem Telefoninterview vorab befragt wurden, ob sie als Softwareentwickler/ innen tätig sind $(n=5149$, davon 1986 Softwareentwickler/innen; nur Letztere wurden in die Studie eingeschlossen und weiter befragt). Insgesamt wurden 8797 Personen im Alter zwischen 18 und 65 Jahren in Bezug auf eine Studienteilnahme kontaktiert, 4166 nahmen an einem computergestützten persönlichen Interview zwischen Dezember 2012 und Juni 2013 teil; die Teilnahmequote betrug $47 \%$ [26]. Von jeder teilnehmenden Person wurde die schriftliche Zustimmung zur Studienteilnahme eingeholt.

Die erste Welle der S-MGA ist ein repräsentativer Querschnitt der sozialversicherungspflichtig Beschäftigten im Alter von 31-60 Jahren. Die Stichprobenziehung erfolgte ebenfalls nach einem zweistufigen Clusterverfahren, allerdings in anderer Form. Zunächst wurden 206 Gemeinden proportional zur Bevölkerungszahl aus $12.227 \mathrm{Ge}$ meinden in Deutschland ausgewählt. Im zweiten Schritt wurden die Studienteilnehmenden zufällig aus den jeweiligen Gemeinden ausgelost. Zur Teilnahme vorgesehen waren alle Arbeitnehmenden in Deutschland, die zwischen 1951 und 1980 geboren sind und zum Stichtag 31. Dezember 2010 sozialversicherungspflichtig beschäftigt waren, d.h. Selbstständige, Beamte und Freiberufler waren ausgeschlossen. Insgesamt wurden 13.590 Adressen aus dem Beschäftigtenregister gezogen. Mit 4549 teilnahmebereiten Beschäftigten wurden zwischen November 2011 und Juni 2012 computergestützte persönliche Interviews von geschulten Interviewern durchgeführt. 38 Interviews waren nicht auswertbar, sodass 4511 Interviews für 
Zbl Arbeitsmed 2021 · 71:8-18 https://doi.org/10.1007/s40664-020-00409-3

(c) Der/die Autor(en) 2020

\section{N. Kersten · M. Formazin · G. Müller}

\section{Burnout und psychosoziale Arbeitsbedingungen in Berufsgruppen mit hohen kognitiven Anforderungen}

\section{Zusammenfassung}

Hintergrund. Burnout ist ein psychologisches Syndrom, das aufgrund seiner Konsequenzen sowohl für die Betroffenen selbst als auch für die Betriebe und die Gesellschaft als Ganzes von hoher Relevanz ist.

Fragestellung. Die Ausprägung von Burnout und sein Zusammenhang mit psychosozialen Arbeitsbedingungen werden in drei Berufsgruppen mit hohen kognitiven Anforderungen - Softwareentwickler/innen, Werbefachleute, Publizist/innen - im Vergleich zur Beschäftigtenpopulation in Deutschland untersucht.

Material und Methoden. Es werden Daten aus zwei Querschnittsstudien zusammengefasst $(n=6553)$. Die psychosozialen Arbeitsbedingungen wurden mit dem Copenhagen Psychosocial Questionnaire (COPSOQ), Burnout mit dem Oldenburger BurnoutInventar (OLBI) erfasst. Um die Beziehung zwischen Arbeitsbedingungen und Burnout zu ermitteln, wurden Regressionsmodelle unter Berücksichtigung von möglichen Confoundern angepasst und zusätzlich Interaktionen zwischen Berufen und Arbeitsbedingungen ermittelt.

Ergebnisse. Die Burnout-Scores sind in den drei Berufsgruppen nicht generell höher als in der Vergleichsgruppe. Werbefachfrauen und Publizistinnen weisen höhere, gegenüber den Softwareentwicklerinnen und der weiblichen Beschäftigtenpopulation, jedoch nicht statistisch signifikante unterschiedliche Burnout-Werte auf. Bei den Männern weisen Softwareentwickler signifikant niedrigere Burnout-Werte als Werbefachmänner auf. Die drei Berufsgruppen haben ein niedrigeres Maß an Führungsqualität und Rollenklarheit sowie ein höheres $\mathrm{Ma} ß$ an quantitativen Anforderungen, aber auch an Entscheidungsspielraum und sozialer Unterstützung als die Beschäftigtenpopulation. Bei Männern und
Frauen sind insbesondere hohe quantitative Anforderungen positiv mit Burnout assoziiert. Arbeitsplatzressourcen, z. B. Führungsqualität, hängen negativ mit Burnout zusammen. Diskussion. Über den Durchschnittseffekt der gesamten Stichprobe hinaus wirken sich insbesondere quantitative Anforderungen, aber auch Führungsqualität und soziale Unterstützung in den drei Berufsgruppen in stärkerem Maße auf das Burnout-Level aus. Die Spezifik der Effekte in den drei Berufsgruppen impliziert, dass Arbeitsgestaltungsmaßnahmen für die jeweiligen Berufsgruppen sowohl allgemeine als auch spezifische Aspekte berücksichtigen sollten.

Schlüsselwörter Erschöpfung · Kognitive Arbeitsanforderungen · Quantitative Arbeitsanforderungen . Copenhagen Psychosocial Questionnaire . Oldenburger Burnout-Inventar

\section{Burnout and psychosocial working conditions in professional groups with high cognitive demands}

\section{Abstract}

Background. Due to its consequences burnout is a psychological syndrome that is of relevance for those affected as well as for companies and society as a whole.

Objective. The extent of burnout and its association with psychosocial working conditions were investigated in three professions with high cognitive demands, i.e. software developers, advertising specialists and publicists, in comparison to the general working population in Germany.

Material and methods. Data from two crosssectional studies were combined $(n=6553)$. Psychosocial working conditions were assessed with the Copenhagen psychosocial questionnaire (COPSOQ) and burnout with the Oldenburg burnout inventory (OLBI). To determine the relationship between working conditions and burnout, regression models were applied considering possible confounders. Additionally, interactions between occupations and working conditions were ascertained.

Results. The burnout scores in the three occupational groups were not generally higher than in the control group. Female advertising professionals and female publicists had higher but not significantly different burnout scores than female software developers and women in the general working population. Among men, software developers had significantly lower burnout values than advertising specialists. The three occupational groups had lower levels of leadership quality and role clarity as well as higher levels of quantitative demands, but also higher decision authority and social support than the general working population. For men and women, particularly high quantitative demands were positively associated with burnout, while job resources, e.g. leadership quality, were negatively associated with burnout.

Conclusion. In the three professions considered both quantitative demands as well as, albeit partly, quality of leadership and social support had a stronger effect on burnout beyond that of the average effect of the general working population. The specificity of the effects in the three professions implies that both generic as well as specific aspects should be taken into consideration for job design measures for each profession.

\section{Keywords}

Exhaustion - Cognitive work demands . Quantitative work demands · Copenhagen Psychosocial Questionnaire · Oldenburg burnout inventory die Analyse verblieben; die Teilnahmequote betrug $36 \%$ [27]. Die schriftlichen Zustimmungen zur Studienteilnahme lagen ebenfalls vor.

\section{Instrumente}

Burnout wurde mit dem Oldenburger Burnout-Inventar (OLBI) erhoben, wobei in den beiden Studien die Subskala für Erschöpfung angewendet wurde, da Erschöpfung als Kernkomponente des
Burnouts verstanden wird $[17,25]$. Die acht Items haben vier Antwortkategorien, die von „stimme voll und ganz

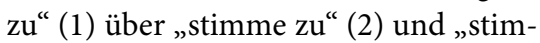
me nicht zu“ (3) bis „stimme überhaupt nicht zu“ (4) reichen. Erschöpfung wird mit Aussagen wie „Es gibt Tage, an 


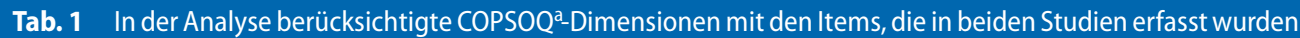

\begin{tabular}{|c|c|}
\hline Dimension & Item-Inhalte \\
\hline \multirow{3}{*}{$\begin{array}{l}\text { Quantitative } \\
\text { Anforderungen }\end{array}$} & Wie häufig müssen Sie sehr schnell arbeiten? \\
\hline & Wie häufig kommt es vor, dass Sie nicht genügend Zeit haben, alle Ihre Aufgaben zu erledigen? \\
\hline & Wie häufig müssen Sie Überstunden machen? \\
\hline \multirow{2}{*}{$\begin{array}{l}\text { Arbeitsplatz- } \\
\text { unsicherheit }\end{array}$} & In welchem Maß machen Sie sich Sorgen, dass Sie arbeitslos werden? \\
\hline & $\begin{array}{l}\text { In welchem Maß machen Sie sich Sorgen, dass es schwierig für Sie wäre, eine neue Arbeit zu finden, wenn Sie arbeitslos wür- } \\
\text { den? }\end{array}$ \\
\hline \multirow[t]{3}{*}{ Rollenklarheit } & Wissen Sie genau, wie weit Ihre Befugnisse bei der Arbeit reichen? \\
\hline & Gibt es klare Ziele für Ihre Arbeit? \\
\hline & Wissen Sie genau, welche Dinge in Ihren Verantwortungsbereich fallen? \\
\hline \multirow{4}{*}{$\begin{array}{l}\text { Entscheidungs- } \\
\text { spielraum }\end{array}$} & Können Sie selbst bestimmen, wann Sie eine Pause machen? \\
\hline & Können Sie mehr oder weniger frei entscheiden, wann Sie Urlaub machen? \\
\hline & Können Sie Ihre Arbeit unterbrechen, um sich mit einem Kollegen zu unterhalten? \\
\hline & Können Sie für Privates Ihren Arbeitsplatz ohne besondere Erlaubnis für eine halbe Stunde verlassen? \\
\hline \multirow[t]{4}{*}{ Führungsqualität } & In welchem Maß sorgt Ihr unmittelbarer Vorgesetzter für gute Entwicklungsmöglichkeiten der einzelnen Mitarbeiter? \\
\hline & In welchem Maß misst Ihr unmittelbarer Vorgesetzter der Arbeitszufriedenheit einen hohen Stellenwert bei? \\
\hline & In welchem Maß plant Ihr unmittelbarer Vorgesetzter die Arbeit gut? \\
\hline & In welchem Maß löst Ihr unmittelbarer Vorgesetzter Konflikte gut? \\
\hline \multirow{3}{*}{$\begin{array}{l}\text { Soziale } \\
\text { Unterstützung } \\
\text { durch Kollegen }\end{array}$} & Wie oft erhalten Sie Hilfe und Unterstützung von Ihren Kollegen? \\
\hline & Wie oft sind Ihre Kollegen bereit, sich Ihre Arbeitsprobleme anzuhören? \\
\hline & Wie oft sprechen Ihre Kollegen mit Ihnen über die Qualität Ihrer Arbeit? \\
\hline \multicolumn{2}{|c|}{$\begin{array}{l}\text { Skalierung der Antwortkategorien beim COPSOQ: } 0-4 \\
\text { Pro COPSOQ-Dimension Berechnung des arithmetischen Mittels über die Antwortskalen } \\
{ }^{a} \text { Copenhagen Psychosocial Questionnaire }\end{array}$} \\
\hline
\end{tabular}

denen ich mich müde fühle, bevor ich zur Arbeit komme“ oder „Nach der Arbeit habe ich genug Energie für meine Freizeitaktivitäten" erfasst. Die Hälfte der Items ist positiv formuliert, die andere Hälfte negativ [11] und erfordert eine umgekehrte Kodierung der entsprechenden Items, wenn der Mittelwert über alle Items berechnet wird. Ein höherer Mittelwert weist auf eine größere Erschöpfung hin [10]. In der S-MGA wurde Erschöpfung mit einem von den Studienteilnehmenden selbst auszufüllenden Fragebogens erfasst, während in der Studie zu Neuroenhancement Erschöpfung im persönlichen Interview erfragt wurde.

Der Copenhagen Psychosocial Questionnaire (COPSOQ) wurde in beiden Studien angewendet, um psychosoziale Arbeitsbedingungen mittels eines computergestützten persönlichen Interviews (CAPI) zu erheben [15]. Erfasst wurden Anforderungen (quantitative Anforderungen, Arbeitsplatzunsicherheit) sowie Ressourcen (Rollenklarheit, Entscheidungsspielraum, Führungsqualität und soziale Unterstützung durch Kollegen) bei der Arbeit (•Tab. 1). Die Antwortkategorien wurden für jedes Item auf einer 5-stufigen Likert-Skala mit Werten zwischen 0 und 4 erhoben und für die Skalenbildung über alle Items gemittelt. Ein höherer COPSOQ-Skalenwert steht für eine hohe Ausprägung der jeweiligen Skala, d.h. bei den quantitativen Anforderungen und der Arbeitsplatzunsicherheit für ungünstige, bei allen anderen Skalen für günstige Arbeitsbedingungen. Die Skalen sind somit so ausgerichtet, dass sich unter der Annahme einer Absenkung des Burnout-Scores bei günstigen Arbeitsbedingungen ein negativer und im gegenteiligen Fall ein positiver Regressionskoeffizient ergibt.

Kognitive Belastungen wurden nur in der Neuroenhancement-Studie über die acht Items B1: 8-14 aus der Langversion des COPSOQ-Fragebogens [20] erhoben, im Sinne der eben berichteten Skalenausrichtung weisen die drei Berufsgruppen im Mittel die Werte 3,17 (Publizist/innen), 3,03 (Werbefachleute) und 2,92 (Softwareentwickler/innen) auf. Ein direkter Vergleich mit der Erwerbspopulation ist nicht möglich, da in S-MGA die- se Subskala nicht erhoben wurde. Nübling und Kollegen [19] geben für Arbeiter einen Score-Wert von 2,16 an, für mittlere Angestellte 2,72 und höhere Angestellte 3,00 (umgerechnet auf die hier verwendete Skale; Originalwerte: 54, 68 und 75), wobei die höheren Angestellten am ehesten den Berufsgruppen entsprechen. Bei einem Anteil von 57,5\% Arbeiter/innen in der S-MGA kann ein insgesamt niedrigerer Skalenmittelwert der kognitiven Belastung in der Vergleichsgruppe vorausgesetzt werden.

\section{Berufsgruppen}

In den Analysen werden vier Gruppen betrachtet: Publizist/innen, Werbefachleute, Softwareentwickler/innen und sozialversicherungspflichtig Beschäftigte als Kontrollgruppe. $\mathrm{Zu}$ den Beschäftigungsbedingungen wurden Angaben zu Arbeitszeit und Nachtarbeit erhoben. Die Arbeitszeit umfasst die wöchentlich geleisteten Arbeitsstunden und wurde in vier Kategorien unterteilt, die zwischen Teilzeit- $(<35 \mathrm{~h})$ und Vollzeitbeschäftigung $(35-40 \mathrm{~h})$ unterscheiden und 


\section{Originalien}

Tab. 2 Deskription der gepoolten Stichprobe (nach Geschlecht)

\begin{tabular}{|c|c|c|}
\hline Variablen & Frauen $(n=2868)^{a}$ & Männer $(n=4020)^{a}$ \\
\hline \multicolumn{3}{|l|}{ Beruf } \\
\hline Publizist/innen $n(\%)$ & $472(17)$ & $536(13)$ \\
\hline Werbefachleute $n(\%)$ & $313(11)$ & $249(6)$ \\
\hline Softwareentwickler/innen $n$ (\%) & $211(7)$ & $1170(29)$ \\
\hline Beschäftigtenpopulation $n$ (\%) & $1872(65)$ & $2065(52)$ \\
\hline \multicolumn{3}{|l|}{ Arbeitsstunden pro Woche } \\
\hline$<35 \mathrm{~h} n(\%)$ & $1039(36)$ & $136(3)$ \\
\hline $35-40 \mathrm{~h} n(\%)$ & $900(32)$ & $1427(36)$ \\
\hline$>40-48 \mathrm{~h} n(\%)$ & $590(21)$ & $1564(39)$ \\
\hline$>48-90 \mathrm{~h} n(\%)$ & $324(11)$ & $864(22)$ \\
\hline \multicolumn{3}{|l|}{ Anzahl der Nachtschichten im Monat } \\
\hline Bei Personen mit Nachtschicht MW (SD) & $4,92(3,8)$ & $5,20(4,2)$ \\
\hline Personen ohne Nachtschicht $n(\%)$ & $2679(78)$ & $3237(70)$ \\
\hline \multicolumn{3}{|l|}{ Alter } \\
\hline$\leq 40$ Jahre $n(\%)$ & $814(28)$ & $1192(30)$ \\
\hline 41-50 Jahre $n(\%)$ & $1132(40)$ & $1546(38)$ \\
\hline$\geq 51$ Jahre $n(\%)$ & $920(32)$ & $1280(32)$ \\
\hline \multicolumn{3}{|l|}{ Allein lebend } \\
\hline Ja $n(\%)$ & $475(17)$ & $683(17)$ \\
\hline Nein $n(\%)$ & $2389(83)$ & $3328(83)$ \\
\hline \multicolumn{3}{|l|}{ Kinder unter 14 Jahren im Haushalt } \\
\hline Ja $n(\%)$ & 785 (27) & $1385(34)$ \\
\hline Nein $n(\%)$ & $2083(73)$ & $2635(66)$ \\
\hline
\end{tabular}

die wöchentliche Höchstarbeitszeit von $48 \mathrm{~h}$ berücksichtigen $(>40-48 \mathrm{~h},>48 \mathrm{~h}$ ). Nachtarbeit wurde mit der mittleren Anzahl von Nachtschichten pro Person und Monat erfasst, Personen ohne Nachtschicht wurde der Wert 0 zugewiesen. Bei der Analyse werden neben den beschäftigungsbezogenen Variablen auch individuelle Merkmale als potenzielle Confounder berücksichtigt: Alter zum Zeitpunkt der Stichprobenziehung ( $\leq 40$ Jahre, 41-50 Jahre und $\geq 51$ Jahre), Kinder unter 14 Jahren im Haushalt, Zusammenleben mit Partner. Berufsoder Schulabschlüsse wurden nicht einbezogen, da keine ausreichende Varianz in den betrachteten Berufsgruppen bestand.

Gemäß ILO-Labour-Force-Konzept wurden in den beiden Studien Personen mit mindestens einer Wochenstunde Arbeitszeit in die Stichprobe aufgenommen. In den hier vorgestellten Analysen wurden Personen mit geringfügiger $\mathrm{Be}$ schäftigung nicht berücksichtigt um $\mathrm{zu}$ gewährleisten, dass die Befragten in einem ausreichenden Maß in das Arbeitsumfeld sozial eingebunden sind. Alle Analysen werden für Männer und Frauen getrennt durchgeführt. Es werden Stichprobenmerkmale und Durchschnittswerte des Burnouts und der psychosozialen Arbeitsbedingungen mit entsprechenden 95\% Konfidenzintervallen (CI) berechnet.

\section{Statistik}

Um den Zusammenhang zwischen psychosozialen Arbeitsbedingungen, Beschäftigungsbedingungen, individuellen Merkmalen und Burnout zu ermitteln, wurden verallgemeinerte lineare Regressionsmodelle berechnet. Die Regressionsanalysen wurden in drei Schritten durchgeführt: Zuerst wurden die Assoziationen zwischen jeder Prädiktorvariablen und Burnout separat geschätzt, wobei die COPSOQ-Skalen als ein Variablenblock behandelt wurden. Im zweiten Schritt wurde ein Gesamtmodell mit allen Variablen unter Ausschluss nicht signifikanter Confounder berechnet. Im dritten Schritt erfolgte eine Erweiterung dieses Ansatzes um Interaktionen zwischen den psychosozialen Arbeitsbedingungen und den Berufen. Die Modelle werden mittels ihrer geschätzten Regressionskoeffizienten $(\widehat{\beta})$ und den zugehörigen $p$-Werten dargestellt. Alle Analysen wurden in SPSS 24 ausgeführt.

\section{Ergebnisse}

Die gepoolte Stichprobe besteht aus 8677 Personen, die zum Stichtag sozialversicherungspflichtig beschäftigt waren. Nach Ausschluss von Ärzt/innen $(n=1195)$, die bereits im Rahmen einer anderen Analyse betrachtet wurden [23], und Personen mit geringfügiger Beschäftigung ( $n=600$; darunter 6 Ärzte mit gelegentlicher oder unregelmäßiger Beschäftigung) verblieben 6888 Beschäftigte für die Analyse, darunter 2868 Frauen $(41,6 \%)$ und 4020 Männer (58,4\%). Bei den Regressionsanalysen entfallen weitere Personen (336 Männer, 


\begin{tabular}{|c|c|c|c|c|}
\hline Variablen & $\begin{array}{l}\text { Publizist/innen } \\
(n=1008)^{\mathrm{a}}\end{array}$ & $\begin{array}{l}\text { Werbefachleute } \\
(n=562)^{a}\end{array}$ & $\begin{array}{l}\text { Softwareentwickler/innen } \\
(n=1381)^{\mathrm{a}}\end{array}$ & $\begin{array}{l}\text { Beschäftigtenpopulation } \\
(n=3937)^{\mathrm{a}}\end{array}$ \\
\hline \multicolumn{5}{|l|}{ Arbeitsstunden pro Woche } \\
\hline$<35 \mathrm{~h} n(\%)$ & $135(14)$ & $69(12)$ & $104(8)$ & $867(22)$ \\
\hline $35-40 \mathrm{~h} n(\%)$ & $289(29)$ & $132(24)$ & $498(36)$ & $1408(36)$ \\
\hline$>40-48 \mathrm{~h} n(\%)$ & $329(33)$ & $190(34)$ & $570(41)$ & $1065(27)$ \\
\hline$>48-90 \mathrm{~h} n(\%)$ & $240(24)$ & $169(30)$ & $208(15)$ & $571(15)$ \\
\hline \multicolumn{5}{|c|}{ Anzahl der Nachtschichten im Monat } \\
\hline $\begin{array}{l}\text { Bei Personen mit Nachtschicht } \\
\text { MW (SD) }\end{array}$ & $4,29(3,7)$ & $4,11(4,4)$ & $3,10(3,5)$ & $6,56(5,1)$ \\
\hline 95 Perzentil & 10,6 & 13,7 & 10,0 & 20,0 \\
\hline $\begin{array}{l}\text { Personen ohne Nachtschicht } n \\
(\%)\end{array}$ & $680(68)$ & $430(76)$ & $1170(85)$ & $3282(83)$ \\
\hline \multicolumn{5}{|l|}{ Alter } \\
\hline$\leq 40$ Jahre $n(\%)$ & $329(33)$ & $320(57)$ & $400(29)$ & $957(24)$ \\
\hline 41-50 Jahre $n(\%)$ & $359(36)$ & $150(27)$ & $545(40)$ & $1624(41)$ \\
\hline$\geq 51$ Jahre $n(\%)$ & $319(32)$ & $91(16)$ & $434(32)$ & $1356(34)$ \\
\hline \multicolumn{5}{|l|}{ Allein lebend } \\
\hline Ja $n(\%)$ & $232(23)$ & $160(29)$ & $233(17)$ & $533(14)$ \\
\hline Nein $n(\%)$ & $772(77)$ & $399(71)$ & $1147(83)$ & $3399(86)$ \\
\hline \multicolumn{5}{|l|}{ Kinder unter 14 Jahren im Haushalt } \\
\hline Ja $n(\%)$ & $332(33)$ & $151(27)$ & $483(35)$ & $1204(31)$ \\
\hline Nein $n(\%)$ & $676(67)$ & $411(73)$ & $898(65)$ & $2733(69)$ \\
\hline
\end{tabular}

322 Frauen) wegen fehlender Werte in verschiedenen Variablen.

Die größte Gruppe in der gepoolten Stichprobe ist die Kontrollgruppe der sozialversicherungspflichtig Beschäftigten $(n=3937)$, gefolgt von Softwareentwickler/innen $(n=1381)$, Publizist/ innen $(n=1008)$ und Werbefachleuten $(n=562)$. Der Geschlechteranteil variiert in den Gruppen, der geringste Frauenanteil findet sich bei den Softwareentwicklern, nur 211 Frauen gehören dieser Berufsgruppe an (- Tab. 2). In der Gesamtstichprobe arbeiten 36,4\% der Frauen in Teilzeit, während dies nur auf 3,4\% der Männer zutrifft. Insgesamt arbeiten $67,9 \%$ der Frauen bis zu $40 \mathrm{~h}$ pro Woche, bei den Männern sind es 39,2 \%. Darüber hinaus arbeiten Männer durchschnittlich mehr Nachtschichten pro Monat. Der Anteil der Männer und Frauen, die ohne Partner leben, ist ähnlich, während etwas mehr Männer als Frauen in der Stichprobe Kinder unter 14 Jahren im Haushalt haben.

In -Tab. 3 werden die soziodemografischen Daten der Studienteilnehmer nach Berufsgruppen wiedergegeben. Da sich die Hauptanalyse auf die Berufsgruppen und deren psychosoziale Arbeitsbedingungen richtet, ist es von Interesse, die Verteilungen der soziodemografischen Daten zwischen zu Berufsgruppen $z u$ vergleichen und die Notwendigkeit möglicher Adjustierungen zu prüfen. Die Vergleichsgruppe weist bei den wöchentlichen Arbeitsstunden im Intervall 35-40 h eine maximale Häufigkeit auf. Bei den Berufsgruppen liegen die Maxima oberhalb von 40 Wochenstunden, wobei die Werbefachleute einen deutlichen Anteil von Personen mit über 48 Wochenstunden aufweisen. Bei der Anzahl der Nachtschichten dominiert die Vergleichsgruppe mit dem höchsten Mittelwert $(6,56)$ und dem höchsten 95 Perzentil (20 Nächte). Dagegen weisen die Publizist/innen und Werbefachleute höhere Anteile an Personen mit Nachtschicht auf. Die Vergleichsgruppe und die Softwareentwickler/innen sind in ihrer Altersverteilung ausgeglichen, die Werbefachleute umfassen besonders viele jüngere Beschäftigte unter 40 Jahren. Damit geht der höchste Anteil an Alleinlebenden und der niedrigste Anteil an
Haushalten mit Kindern unter 14 Jahren einher.

Für die Gesamtstichprobe liegt der durchschnittliche Burnout-Score bei 2,28 (95\% CI 2,26-2,29) und ist bei Frauen (MW $=2,32$; 95\% CI 2,30-2,34) signifikant höher als bei Männern $(\mathrm{MW}=2,24$; 95\% CI 2,23-2,26). In - Tab. 4 sind die Mittelwerte der Burnout- und COPSOQSkalen mit entsprechenden 95\% CI für die Berufsgruppen und die Kontrollgruppe aufgeführt. Werbefachfrauen und Publizistinnen weisen unter den Frauen die höchsten Burnout-Scores auf, unterscheiden sich jedoch nicht statistisch signifikant von den Softwareentwicklerinnen oder den sozialversicherungspflichtig beschäftigten Frauen. Werbefachmänner haben die höchsten Burnout-Scores und liegen signifikant über den Softwareentwicklern (Disjunktheit der Konfidenzintervalle), Letztere liegen signifikant unter der Beschäftigtenpopulation.

Frauen weisen bei fast allen COPSOQ-Skalen in den drei Berufsgruppen ungünstigere Score-Werte auf als Männer, wenngleich diese Unterschiede sta- 
Tab. 4 Mittelwerte und zugehörige 95\% Konfidenzintervalle sowie Standardabweichungen des OLB| ${ }^{\mathrm{a}}$ und der psychosozialen Arbeitsbedingungen (COPSOQ $^{\mathrm{b}}$ )

\begin{tabular}{|c|c|c|c|c|c|c|c|c|}
\hline & \multicolumn{2}{|c|}{ Publizist/innen } & \multicolumn{2}{|c|}{ Werbefachleute } & \multicolumn{2}{|c|}{ Softwareentwickler/innen } & \multicolumn{2}{|c|}{ Beschäftigtenpopulation } \\
\hline & Frauen & Männer & Frauen & Männer & Frauen & Männer & Frauen & Männer \\
\hline & $\begin{array}{l}\text { MW }(95 \% \\
\text { Cl) }\end{array}$ & $\begin{array}{l}\text { MW }(95 \% \\
\text { Cl) }\end{array}$ & $\begin{array}{l}\text { MW }(95 \% \\
\text { Cl) }\end{array}$ & $\begin{array}{l}\text { MW }(95 \% \\
\text { Cl) }\end{array}$ & $\begin{array}{l}\text { MW }(95 \% \\
\text { Cl) }\end{array}$ & $\begin{array}{l}\text { MW }(95 \% \\
\text { CI) }\end{array}$ & $\begin{array}{l}\text { MW }(95 \% \\
\text { Cl) }\end{array}$ & $\begin{array}{l}\text { MW }(95 \% \\
\text { Cl) }\end{array}$ \\
\hline OLBI-Skala & $\begin{array}{l}2,38 \\
(2,33-2,42)\end{array}$ & $\begin{array}{l}2,24 \\
(2,19-2,28)\end{array}$ & $\begin{array}{l}2,38 \\
(2,31-2,44)\end{array}$ & $\begin{array}{l}2,35 \\
(2,28-2,42)\end{array}$ & $\begin{array}{l}2,27 \\
(2,20-2,34)\end{array}$ & $\begin{array}{l}2,17 \\
(2,14-2,20)\end{array}$ & $\begin{array}{l}2,31 \\
(2,29-2,34)\end{array}$ & $\begin{array}{l}2,29 \\
(2,26-2,31)\end{array}$ \\
\hline \multicolumn{9}{|l|}{ COPSOQ-Skalen } \\
\hline $\begin{array}{l}\text { Quantitative Anfor- } \\
\text { derungen }\end{array}$ & $\begin{array}{l}2,61 \\
(2,54-2,67)\end{array}$ & $\begin{array}{l}2,56 \\
(2,50-2,62)\end{array}$ & $\begin{array}{l}2,63 \\
(2,55-2,71)\end{array}$ & $\begin{array}{l}2,57 \\
(2,48-2,67)\end{array}$ & $\begin{array}{l}2,39 \\
(2,29-2,49)\end{array}$ & $\begin{array}{l}2,28 \\
(2,24-2,32)\end{array}$ & $\begin{array}{l}2,19 \\
(2,15-2,23)\end{array}$ & $\begin{array}{l}2,21 \\
(2,18-2,25)\end{array}$ \\
\hline $\begin{array}{l}\text { Arbeitsplatz- } \\
\text { unsicherheit }\end{array}$ & $\begin{array}{l}1,71 \\
(1,62-1,80)\end{array}$ & $\begin{array}{l}1,58 \\
(1,50-1,66)\end{array}$ & $\begin{array}{l}1,49 \\
(1,38-1,60)\end{array}$ & $\begin{array}{l}1,41 \\
(1,29-1,53)\end{array}$ & $\begin{array}{l}1,63 \\
(1,49-1,77)\end{array}$ & $\begin{array}{l}1,22 \\
(1,16-1,27)\end{array}$ & $\begin{array}{l}1,50 \\
(1,45-1,56)\end{array}$ & $\begin{array}{l}1,45 \\
(1,40-1,50)\end{array}$ \\
\hline Rollenklarheit & $\begin{array}{l}3,10 \\
(3,04-3,16)\end{array}$ & $\begin{array}{l}3,18 \\
(3,13-3,24)\end{array}$ & $\begin{array}{l}2,98 \\
(2,90-3,06)\end{array}$ & $\begin{array}{l}3,10 \\
(3,02-3,18)\end{array}$ & $\begin{array}{l}2,98 \\
(2,89-3,06)\end{array}$ & $\begin{array}{l}3,00 \\
(2,97-3,05)\end{array}$ & $\begin{array}{l}3,32 \\
(3,30-3,35)\end{array}$ & $\begin{array}{l}3,25 \\
(3,22-3,28)\end{array}$ \\
\hline $\begin{array}{l}\text { Entscheidungs- } \\
\text { spielraum }\end{array}$ & $\begin{array}{l}2,82 \\
(2,75-2,89)\end{array}$ & $\begin{array}{l}2,93 \\
(2,87-3,00)\end{array}$ & $\begin{array}{l}2,77 \\
(2,67-2,87)\end{array}$ & $\begin{array}{l}2,87 \\
(2,76-2,97)\end{array}$ & $\begin{array}{l}3,11 \\
(3,01-3,20)\end{array}$ & $\begin{array}{l}3,22 \\
(3,18-3,25)\end{array}$ & $\begin{array}{l}2,02 \\
(1,97-2,08)\end{array}$ & $\begin{array}{l}2,41 \\
(2,37-2,46)\end{array}$ \\
\hline Führungsqualität & $\begin{array}{l}1,82 \\
(1,74-1,91)\end{array}$ & $\begin{array}{l}2,01 \\
(1,93-2,09)\end{array}$ & $\begin{array}{l}1,98 \\
(1,89-2,08)\end{array}$ & $\begin{array}{l}2,05 \\
(1,94-2,16)\end{array}$ & $\begin{array}{l}2,19 \\
(2,08-2,30)\end{array}$ & $\begin{array}{l}2,22 \\
(2,17-2,26)\end{array}$ & $\begin{array}{l}2,34 \\
(2,29-2,39)\end{array}$ & $\begin{array}{l}2,24 \\
(2,20-2,28)\end{array}$ \\
\hline $\begin{array}{l}\text { Soziale Unterstüt- } \\
\text { zung }\end{array}$ & $\begin{array}{l}2,61 \\
(2,54-2,68)\end{array}$ & $\begin{array}{l}2,63 \\
(2,57-2,69)\end{array}$ & $\begin{array}{l}2,64 \\
(2,55-2,73)\end{array}$ & $\begin{array}{l}2,62 \\
(2,53-2,71)\end{array}$ & $\begin{array}{l}2,68 \\
(2,58-2,78)\end{array}$ & $\begin{array}{l}2,67 \\
(2,63-2,71)\end{array}$ & $\begin{array}{l}2,56 \\
(2,52-2,60)\end{array}$ & $\begin{array}{l}2,55 \\
(2,51-2,59)\end{array}$ \\
\hline
\end{tabular}

tistisch nicht signifikant sind; in der Kontrollgruppe tritt ein solches Muster nicht auf. Im Vergleich zu den sozialversicherungspflichtig Beschäftigten weisen die Beschäftigten der drei Berufsgruppen geringere Werte bei der Rollenklarheit und der Führungsqualität sowie höhere Werte bei den quantitativen Anforderungen auf. Beim Entscheidungsspielraum und bei der sozialen Unterstützung haben sie höhere Werte als die Kontrollgruppe. Bezüglich der Arbeitsplatzunsicherheit ist das Muster uneinheitlich.

In der ersten Regressionsanalyse wurden die Beziehungen zwischen jeder Prädiktorvariablen und Burnout separat geschätzt (•Tab. 5; Modell 1). Nur die Publizistinnen $(\widehat{\beta}=0,060 ; p<0,01)$ weisen signifikant höhere Burnout-Scores auf als Frauen in der Kontrollgruppe. Bei den Männern finden sich niedrigere Burnout-Scores bei Softwareentwicklern $(\widehat{\beta}=-0,119 ; p<0,001)$. Die psychosozialen Arbeitsbedingungen sind alle signifikant mit Burnout verbunden. Mit zunehmenden Ressourcen bei der Arbeit sind niedrigere Burnout-Scores bei Männern und Frauen zu beobachten, während höhere quantitative Anforderungen und Arbeitsplatzunsicherheit zu höheren Werten führen. Die stärkste Assoziation besteht bei quantitativen
Anforderungen: Ein Anstieg um den Wert 1 auf der COPSOQ-Subskala führt $\mathrm{zu}$ einem Zuwachs von 0,239 $(p<0,001$; Frauen) bzw. 0,199 ( $p<0,001$; Männer) bei den Burnout-Scores. Arbeitszeiten von mehr als $40 \mathrm{~h}$ und die Anzahl der Nachtschichten gehen mit einem höheren Burnout-Level einher. Ebenso finden sich höhere Burnout-Scores bei den 41-50 Jahre alten Männern ( $\widehat{\beta}=$ 0,068; $p<0,05)$ in Relation zur Gruppe mit 40 Jahren und jünger. Das Leben ohne Partner und mit Kindern unter 14 Jahren ist nur bei Frauen signifikant mit Burnout verbunden, wobei Frauen ohne Partner höhere $(\widehat{\beta}=0,130$; $p<0,001)$ und Frauen mit Kindern niedrigere Werte $(\widehat{\beta}=-0,109 ; p<0,001)$ aufweisen.

In der zweiten Regressionsanalyse wurden die adjustierten Effekte zwischen den Prädiktorvariablen und Burnout geschätzt (• Tab. 5, Modell 2). Das heißt, es wurde ein Gesamtmodell berechnet, das alle Prädiktoren einschließt, die nach einer Modellreduzierung einen signifikanten Bezug zum Burnout aufweisen. In diesem Modell ist Burnout nur mit den Arbeitsfaktoren assoziiert, während die personenbezogenen Faktoren keine über die Arbeitsfaktoren hinausgehende Wirkung haben, das bedeutet, sie erklären keine Varianz im Outcome, die sich nicht schon durch die Arbeitsfaktoren erklären lässt. Quantitative Anforderungen weisen nach wie vor den stärksten Zusammenhang zum Burnout auf (Frauen: $\widehat{\beta}=0,228 ; p<0,001$; Männer: $\widehat{\beta}=0,210 ; p<0,001)$. Die Arbeitsstunden pro Wochen haben nur noch bei einer Arbeitszeit von weniger als $35 \mathrm{~h}$ einen signifikanten Effekt, bei den Frauen liegt hier der BurnoutScore signifikant niedriger als bei Vollzeitarbeit (35-40h), bei den Männern signifikant höher. Eine steigende Zahl von Nachtschichten hängt bei Frauen, nicht jedoch bei Männern mit einem steigenden Burnout-Level zusammen.

Die Regressionskoeffizienten der psychosozialen Arbeitsbedingungen bleiben von Modell 1 zu Modell 2 in Richtung und Höhe vergleichsweise konstant, die Effekte der Berufsgruppen unterliegen dagegen erheblichen Veränderungen: Bei den Publizistinnen kehrt sich der Effekt um, bei den Publizisten und den Softwareentwicklern ändert sich die Höhe der Assoziation und der Effekt wird bei Erstgenannten statistisch signifikant. Um die Frage zu klären, in welchem Verhältnis Effekte der Berufsgruppen und Arbeitsbedingungen stehen, wurde in einer ergänzenden Analyse Modell 2 um In- 
Tab. 5 Verallgemeinerte lineare Modelle mit der Zielvariable OLB|a-Skala: Regressionskoeffizienten und zugehörige $p$-Werte: Modell 1: Assoziation zwischen jeder Prädiktorvariable und der OLBI-Skala separat. Modell 2: Assoziation zwischen den im Gesamtmodell signifikanten Prädiktorvariablen und der OLBI-Skala. Modell 3: Modell 2+Interaktionsterme

\begin{tabular}{|c|c|c|c|c|c|c|}
\hline \multirow{3}{*}{ Unabhängige Variablen } & \multicolumn{3}{|c|}{ Frauen $(n=2546)$} & \multicolumn{3}{|c|}{ Männer ( $n=3664)$} \\
\hline & Modell 1 & Modell 2 & Modell 3 & Modell 1 & Modell 2 & Modell 3 \\
\hline & $\widehat{\beta}$ & $\widehat{\beta}$ & $\widehat{\beta}$ & $\widehat{\beta}$ & $\widehat{\beta}$ & $\widehat{\beta}$ \\
\hline \multicolumn{7}{|l|}{ Berufe } \\
\hline Publizist/innen & $0,060^{*}$ & $-0,065^{*}$ & 0,109 & $-0,050$ & $-0,116^{* * *}$ & $-0,110$ \\
\hline Werbefachleute & 0,064 & $-0,053$ & $-0,013$ & 0,068 & 0,003 & $-0,215$ \\
\hline Softwareentwickler/innen & $-0,047$ & $-0,049$ & 0,192 & $-0,119^{* * *}$ & $-0,066^{* * *}$ & $-0,014$ \\
\hline Beschäftigtenpopulation & 0 & 0 & 0 & 0 & 0 & 0 \\
\hline \multicolumn{7}{|c|}{ Psychosoziale Arbeitsbedingungen } \\
\hline Quantitative Anforderungen & $0,239^{* * *}$ & $0,228^{* * * *}$ & $0,214^{* * *}$ & $0,199^{* * *}$ & $0,210^{* * *}$ & $0,176^{* * *}$ \\
\hline Arbeitsplatzunsicherheit & $0,071^{* * *}$ & $0,071^{* * *}$ & $0,070^{* * *}$ & $0,074^{* * *}$ & $0,075^{* * *}$ & $0,073^{* * *}$ \\
\hline Rollenklarheit & $-0,074^{* * *}$ & $-0,081^{* * *}$ & $-0,076^{* * *}$ & $-0,099 * * *$ & $-0,104^{* * *}$ & $-0,102^{* * *}$ \\
\hline Entscheidungsspielraum & $-0,096^{* * *}$ & $-0,088^{* * *}$ & $-0,089^{* * *}$ & $-0,105^{* * *}$ & $-0,088^{* * *}$ & $-0,086^{* * *}$ \\
\hline Führungsqualität & $-0,086^{* * *}$ & $-0,088^{* * *}$ & $-0,079^{* * *}$ & $-0,102^{* * *}$ & $-0,104^{* * *}$ & $-0,090^{* * *}$ \\
\hline $\begin{array}{l}\text { Soziale Unterstützung durch } \\
\text { Kollegen }\end{array}$ & $-0,060^{* * *}$ & $-0,058^{* * *}$ & $-0,035^{* *}$ & $-0,051^{* * *}$ & $-0,048^{* * *}$ & $-0,028^{*}$ \\
\hline \multicolumn{7}{|l|}{ Interaktionen } \\
\hline \multicolumn{7}{|l|}{ Quantitative Anforderungen } \\
\hline Publizist/innen & - & - & 0,025 & - & - & $0,069^{* *}$ \\
\hline Werbefachleute & - & - & $0,093^{*}$ & - & - & $0,113^{* *}$ \\
\hline Softwareentwickler/innen & - & - & 0,011 & - & - & $0,075^{* *}$ \\
\hline \multicolumn{7}{|l|}{ Führungsqualität } \\
\hline Publizist/innen & - & - & 0,013 & - & - & $-0,068^{*}$ \\
\hline Werbefachleute & - & - & $-0,102^{* *}$ & - & - & $-0,075^{*}$ \\
\hline Softwareentwickler/innen & - & - & $-0,020$ & - & - & 0,007 \\
\hline \multicolumn{7}{|c|}{ Soziale Unterstützung durch Kollegen } \\
\hline Publizist/innen & - & - & $-0,096^{* *}$ & - & - & $-0,012$ \\
\hline Werbefachleute & - & - & $-0,027$ & - & - & 0,036 \\
\hline Softwareentwickler/innen & - & - & $-0,081$ & - & - & $-0,090^{* * *}$ \\
\hline \multicolumn{7}{|l|}{ Arbeitsstunden pro Woche } \\
\hline$<35 \mathrm{~h}$ & $-0,111^{* * *}$ & $-0,098^{* * *}$ & $-0,102^{* * *}$ & $0,144^{* *}$ & $0,131^{* *}$ & $0,140^{* *}$ \\
\hline $35-40$ & 0 & 0 & 0 & 0 & 0 & 0 \\
\hline$>40-48$ & $0,064^{*}$ & $-0,011$ & $-0,012$ & $0,093^{* * *}$ & $-0,004$ & $-0,007$ \\
\hline$>48-90$ & $0,252^{* * *}$ & 0,039 & 0,022 & $0,181^{* * *}$ & $-0,012$ & $-0,021$ \\
\hline \multicolumn{7}{|c|}{ Anzahl der Nachtschichten im Monat } \\
\hline- & $0,024^{* * *}$ & $0,010^{* *}$ & $0,010^{* *}$ & $0,011^{* * *}$ & 0,003 & 0,002 \\
\hline \multicolumn{7}{|l|}{ Alter } \\
\hline$\leq 40$ Jahre & 0 & - & - & 0 & - & - \\
\hline 41-50 Jahre & $-0,002$ & - & - & $0,068^{*}$ & - & - \\
\hline$\geq 51$ Jahre & 0,031 & - & - & 0,015 & - & - \\
\hline \multicolumn{7}{|l|}{ Allein lebend } \\
\hline $\mathrm{Ja}$ & $0,130^{* * *}$ & - & - & 0,037 & - & - \\
\hline Nein & 0 & - & - & 0 & - & - \\
\hline \multicolumn{7}{|c|}{ Kinder unter 14 Jahren im Haushalt } \\
\hline Ja & $-0,109^{* * *}$ & - & - & $-0,027$ & - & - \\
\hline Nein & 0 & - & - & 0 & - & - \\
\hline
\end{tabular}


teraktionsterme von psychosozialen Arbeitsbedingungen und Berufsgruppe erweitert. Von sechs einbezogenen Interaktionstermen waren die der quantitativen Anforderungen, der Führungsqualität und der sozialen Unterstützung durch Kollegen signifikant. Modell 3 beinhaltet eine Erweiterung von Modell 2 um diese drei Interaktionsterme. Statistisch signifikant sind bei den Männern die kombinierten Effekte von sozialer Unterstützung durch die Kollegen bei den Softwareentwicklern $(\widehat{\beta}=-0,090 ; p<0,001)$ sowie der Führungsqualität bei den $\mathrm{Pu}$ blizisten $(\widehat{\beta}=-0,068 ; p<0,05)$ und den Werbefachleuten $(\widehat{\beta}=-0,075 ; p<0,05)$, bei den Frauen sind es die kombinierten Effekte von sozialer Unterstützung durch die Kollegen bei den Publizistinnen $(\widehat{\beta}=$ $-0,096 ; p<0,01)$ und der Führungsqualität bei den Werbefachfrauen $(\widehat{\beta}=-0,102$; $p<0,01)$. Des Weiteren gibt es bei den quantitativen Anforderungen über die Haupteffekte hinausgehende, den Burnout-Score erhöhende Interaktionseffekte bei allen drei betrachteten Berufsgruppen bei den Männern, bei den Frauen betrifft dies nur die Werbefachfrauen. Die Regressionskoeffizienten für die psychosozialen Arbeitsbedingungen werden in diesem Modell 3 in ihrem Betrag etwas geringer, dazu addieren sich die berufsspezifischen Effekte der Interaktionsterme. Es gibt keine signifikant von Null verschiedenen Berufseffekte mehr.

\section{Diskussion}

Burnout und sein Zusammenhang zu psychosozialen Arbeitsbedingungen wurden in einer gepoolten Stichprobe aus drei Berufsgruppen, die sich durch hohe kognitive Belastungen auszeichnen, im Vergleich zu einer Referenzgruppe aus der Beschäftigtenpopulation Deutschlands untersucht. Bei den Burnout-Scores bestehen signifikante Unterschiede zwischen den untersuchten Berufsgruppen und der Beschäftigtenpopulation (•Tab. 4). Während bei weiblichen Werbefachleuten und Publizistinnen im Vergleich zur weiblichen Beschäftigtenpopulation höhere Burnout-Scores festgestellt wurden, die im Regressionsmodell allerdings nur bei den Publizistinnen als statisch signifi- kant ausgewiesen werden (Modell 1), sind diese bei männlichen Softwareentwicklern im Vergleich zur männlichen Beschäftigtenpopulation geringer, was in Modell 1 bestätigt wird.

Hohe quantitative Anforderungen bei der Arbeit und Arbeitsplatzunsicherheit sind mit höheren BurnoutScores verbunden, wobei quantitative Anforderungen in unserer Stichprobe den stärksten Zusammenhang mit Burnout haben und damit im Einklang mit Ergebnissen früherer Untersuchungen stehen, die nicht auf die drei Berufsgruppen beschränkt waren $[5,22,28]$. Arbeitsplatzressourcen wie Rollenklarheit, Entscheidungsspielraum, die Führungsqualität des Vorgesetzten und die soziale Unterstützung durch die Kollegen sind negativ mit Burnout verbunden und erscheinen demzufolge protektiv für Burnout. Da die drei betrachteten Berufsgruppen einerseits höhere quantitative Anforderungen als die Beschäftigtenpopulation berichten, andererseits aber auch einen höheren Entscheidungsspielraum, deuten die Ergebnisse darauf hin, dass der hohe Entscheidungsspielraum den Effekt der hohen quantitativen Anforderungen auf die Burnout-Scores teilweise abfedern kann.

Frauen mit Teilzeitbeschäftigung $(<35$ Arbeitsstunden pro Woche), die mit $36 \%$ eine recht große Gruppe sind, weisen niedrigere Burnout-Scores auf als Frauen in Vollzeitbeschäftigung. Dies deckt sich insgesamt mit Befunden aus dem Arbeitszeitreport Deutschland 2016, wonach Beschäftigte in kurzer Teilzeit (unter $20 \mathrm{~h}$ ) am wenigsten Beschwerden berichten [30]. Denkbar ist, dass Frauen in Teilzeit tätig sind, um den verschiedenen Anforderungen des Arbeits- und Privatlebens gerecht zu werden [7]. Dies lässt sich auf Basis der vorliegenden Daten nicht näher untersuchen, da weitere Anforderungen für die Berufsgruppen, wie bspw. Pflegetätigkeiten, nicht erfragt wurden. Im Gegensatz dazu wurde bei Männern, die in Teilzeit arbeiten, höhere Burnout-Scores festgestellt als bei Männern in Vollzeitbeschäftigung. Allerdings handelt es sich dabei um eine sehr kleine Gruppe (3\% der männlichen Stichprobe). In der S-MGA, die die Kontrollgruppe in der vorliegenden Analy- se bildet, wurde neben anderen Variablen auch der Gesundheitszustand der Beschäftigten mittels der norwegischen Funktionsskala gemessen, die Werte zwischen 1 und 5 annehmen kann, wobei geringe Werte für eine hohe Funktionsfähigkeit stehen [14]. Für vollzeitbeschäftigte Männer ergibt sich hier ein Skalenmittelwert von 1,15 , für in Teilzeit beschäftigte Männer mit einer Arbeitszeit zwischen 14 und $34 \mathrm{~h}$ ein Skalenmittelwert von 1,20. Dies zeigt, dass Männer mit einer Teilzeitbeschäftigung einen leicht schlechteren Gesundheitszustand berichten als solche in Vollzeitbeschäftigung. Das Ergebnis ist konform mit einer Analyse von Daten der Techniker Krankenkasse aus dem Jahr 2013 [13], bei der Männer in Teilzeitarbeit längere Arbeitsunfähigkeitszeiten bei psychischen Störungen aufweisen als Männer in Vollzeitarbeit, während bei den Frauen die umgekehrte Relation auftritt. Die Ergebnisse zu Burnout in der vorliegenden Analyse sind vergleichbar, so dass die Vermutung naheliegt, dass Gesundheitsaspekte bei Männern ein wesentliches Motiv für Teilzeitarbeit sind.

Nach Kenntnisstand der Autoren ist dies die erste Studie zum Zusammenhang zwischen psychosozialen Arbeitsbedingungen und Burnout, die auf einer bundesweit repräsentativen Stichprobe der Beschäftigtenpopulation und einer großen Stichprobe von drei Berufsgruppen, die sich durch eine hohe kognitive Belastung auszeichnen, in Deutschland basiert. Dies stellt eine Stärke der Studie dar.

Die vorliegende Studie weist einige Einschränkungen auf. Beide Teildatensätze beruhen auf Querschnittstudien, so dass keine Rückschlüsse auf Kausalzusammenhänge möglich sind. Dass die Zusammenhänge zwischen hohen quantitativen Arbeitsanforderungen und emotionaler Erschöpfung nicht nur das Ergebnis einer negativ veränderten Wahrnehmung der Arbeitssituation darstellen, stützen Befunde aus Längsschnittstudien [29]. Das gewählte Stichprobendesign schloss nur sozialversicherungspflichtige Beschäftigte ein, Beamte, Selbstständige und Freiberufler waren ausgenommen. Diese Einschränkung ist allerdings nicht als 
schwerwiegend $\mathrm{zu}$ bewerten, da viele Fachkräfte aus den drei Berufsgruppen sozialversicherungspflichtig Beschäftigte sind, so dass die Datenbasis der S-MGAStichprobe als Vergleichsgruppe eine geeignete Wahl ist. Geringfügige Überschneidungen zwischen den Stichproben sind möglich, so gibt es in der S-MGAStichprobe insgesamt 43 Publizist/innen, Werbefachleute und Softwareentwickler/innen unter den insgesamt 3937 Befragten, deren Daten in die Analyse einflossen. Dies dürfte die Ergebnisse dieser Arbeit nicht beeinflussen.

Burnout wurde in der vorliegenden Untersuchung auf Basis der Erschöpfungsskala, nicht des kompletten OLBI, ermittelt, da Erschöpfung als Kernkomponente des Burnouts angesehen wird [17]. Es gibt Unterschiede zwischen den beiden gepoolten Stichproben in der Erhebungsmethode des Burnouts, was die Analyseergebnisse beeinflusst haben könnte. Bei S-MGA wurde Burnout mit einem selbstauszufüllenden Fragebogen in Abwesenheit des Interviewers ermittelt, während in der Studie zum Neuroenhancement die Daten zum Burnout während eines persönlichen Interviews erhoben wurden. Denkbar wäre, dass die Befragten im Interview aufgrund des direkten Kontakts stärker sozial erwünscht geantwortet haben. Die Ergebnisse weisen jedoch nicht darauf hin, dass ein Antwortverhalten in Richtung sozialer Erwünschtheit zu einer Unterschätzung des Burnouts in den drei Berufsgruppen geführt hat, da die Burnout-Werte in den drei Berufsgruppen nicht durchgehend niedriger sind als in der Beschäftigtenpopulation.

Zusammenfassend lässt sich sagen, dass Beschäftigte in Berufen, die durch hohe kognitive Belastungen gekennzeichnet sind, im Vergleich zur Beschäftigtenpopulation ein erkennbares Muster psychosozialer Arbeitsbedingungen aufweisen, welches sich insbesondere durch hohe quantitative Anforderungen und gleichzeitig einen hohen Entscheidungsspielraum auszeichnet. Burnout ist vorrangig mit den psychosozialen Arbeitsbedingungen assoziiert und in Relation zur Beschäftigtenpopulation nur bedingt mit den drei betrachteten Berufsgruppen. Statt eines allgemeinen
Effektes des Berufes auf den BurnoutSkalenwert gibt es einzelne Kombinationseffekte, die stressorenseitig vorrangig die quantitativen Anforderungen betreffen und auf Seiten der Ressourcen die Führungsqualität und die soziale Unterstützung durch die Kollegen. Die quantitativen Anforderungen erhöhen in den Berufsgruppen den Burnout-Score über den Durchschnittseffekt der gesamten Stichprobe hinaus, die Ressourcen senken ihn ab.

\section{Fazit für die Praxis}

- In Berufsgruppen mit hohen kognitiven Anforderungen lässt sich ein Muster der psychosozialen Arbeitsbedingungen erkennen, welches im Vergleich zur Beschäftigtenpopulation durch eine geringere Führungsqualität und Rollenklarheit sowie höhere quantitative Anforderungen geprägt ist, aber auch einen höheren Entscheidungsspielraum und eine höhere soziale Unterstützung.

- Bezogen auf die betrachteten Berufsgruppen ist herauszustellen, dass in diesen quantitativen Anforderungen sowie Entscheidungsspielraum und soziale Unterstützung jeweils stärkere Effekte haben als in der Beschäftigtenpopulation.

- Die Ableitung von Maßnahmen der Arbeitsgestaltung sollte neben der Berücksichtigung von branchenund tätigkeitsübergreifenden relevanten Schlüsselfaktoren weitere berufsgruppenspezifische Aspekte in den Blick nehmen. Dies steht im Einklang mit den Empfehlungen der Gemeinsamen Deutschen Arbeitsschutzstrategie (GDA) zur Umsetzung der Gefährdungsbeurteilung psychischer Belastung.

\section{Korrespondenzadresse}

Dr. rer. nat. Norbert Kersten

Fachbereich Arbeit und Gesundheit,

Bundesanstalt für Arbeitsschutz und Arbeitsmedizin

Nöldnerstr. 40-42, 10317 Berlin, Deutschland

kersten.norbert@baua.bund.de

Funding. Open Access funding enabled and organized by Projekt DEAL.
Einhaltung ethischer Richtlinien

Interessenkonflikt. N. Kersten, M. Formazin und G. Müller geben an, dass kein Interessenkonflikt besteht.

Für diesen Beitrag wurden von den Autoren keine Studien an Menschen oder Tieren durchgeführt. Für die aufgeführten Studien gelten die jeweils dort angegebenen ethischen Richtlinien.

Open Access. Dieser Artikel wird unter der Creative Commons Namensnennung 4.0 International Lizenz veröffentlicht, welche die Nutzung, Vervielfältigung, Bearbeitung, Verbreitung und Wiedergabe in jeglichem Medium und Format erlaubt, sofern Sie den/die ursprünglichen Autor(en) und die Quelle ordnungsgemäß nennen, einen Link zur Creative Commons Lizenz beifügen und angeben, ob Änderungen vorgenommen wurden.

Die in diesem Artikel enthaltenen Bilder und sonstiges Drittmaterial unterliegen ebenfalls der genannten Creative Commons Lizenz, sofern sich aus der Abbildungslegende nichts anderes ergibt. Sofern das betreffende Material nicht unter der genannten Creative Commons Lizenz steht und die betreffende Handlung nicht nach gesetzlichen Vorschriften erlaubt ist, ist für die oben aufgeführten Weiterverwendungen des Materials die Einwilligung des jeweiligen Rechteinhabers einzuholen.

Weitere Details zur Lizenz entnehmen Sie bitte der Lizenzinformation auf http://creativecommons.org/ licenses/by/4.0/deed.de.

\section{Literatur}

1. Ahola K, Gould R, Virtanen M et al (2009) Occupational burnout as a predictor of disability pension: a population-based cohort study. Occup Environ Med 66:284-290

2. Ahola K, Hakanen J (2007) Job strain, burnout, and depressive symptoms: a prospective study among dentists. J Affect Disord 104:103-110

3. Ahola K, Honkonen T, Isometsa E et al (2005) The relationship between job-related burnout and depressive disorders - results from the Finnish health 2000 study. J Affect Disord 88:55-62

4. Ahola K, Salminen S, Toppinen-Tanner S et al (2013) Occupational burnout and severe injuries: an eight-year prospective cohort study among Finnish forest industry workers. Sangyo Eiseigaku Zasshi 55:450-457

5. Aronsson G, Theorell T, Grape T et al (2017) A systematic review including meta-analysis of work environment and burnout symptoms. BMC Public Health 17:264

6. Bakker AB, Demerouti E, Verbeke W (2004) Using the job demands-resources model to predict burnout and performance. Hum Resour Manage 43:83-104

7. BMAS (2014) Sicherheit und Gesundheit bei der Arbeit 2013 - Unfallverhütungsbericht Arbeit. Bundesanstalt für Arbeitsschutz und Arbeitsmedizin, Dortmund, Berlin, Chemnitz

8. Borritz M, Bultmann U, Rugulies R et al (2005) Psychosocial work characteristics as predictors for burnout: findings from 3-year follow up of the PUMA study. J Occup Environ Med 47:1015-1025 
9. Demerouti E, Bakker AB, Nachreiner F et al (2001) The job demands-resources model of burnout. JAppl Psychol 86:499-512

10. Demerouti E, Mostert K, Bakker AB (2010) Burnout and work engagement: $a$ thorough investigation of the independency of both constructs. J Occup Health Psychol 15:209-222

11. Demerouti E, Nachreiner F (1998) Zur Spezifität von Burnout für Dienstleistungsberufe: Fakt oder Artefakt.ZArb Wiss 52:82-89

12. Dragano N, Siegrist J, Wahrendorf M (2011) Welfare regimes, labour policies and unhealthy psychosocial working conditions: a comparative study with 9917 older employees from 12 European countries. J Epidemiol Community Health 65:793-799

13. Grobe TG (2016) Teilzeitarbeit und Gesundheit von Männern : Ergebnisse auf der Basis von Routinedaten einer Krankenkasse. Bundesgesundheitsblatt Gesundheitsforschung Gesundheitsschutz 59:942-949

14. Jankowiak S, Rose U, Kersten N (2018) Application of the ICF based Norwegian function assessment scale to employees in Germany. J Occup Med Toxicol 13:3-3

15. Kristensen T, Hannerz $H$, Hogh A et al (2005) The Copenhagen psychosocial questionnaire a tool for the assessment and improvement of the psychosocial work environment. Scand J Work Environ Health 31:438-449

16. Leiter MP, Hakanen J, Ahola K et al (2012) Organizational predictors and health consequences of changes in burnout: a 12-year cohort study. JOrganiz Behav 34:959-973

17. Maslach C, Jackson SE, Leiter MP (1997) Maslach burnout inventory. In: Zalaquett CP, Wood RJ(Hrsg) Evaluating stress: a book of resources, 3. Aufl. Scarecrow Press, London, S 191-218

18. Müller G, Freude G, Kersten N (2019) Neuroenhancement in Deutschland am Beispiel von vier Berufsgruppen. Gesundheitswesen 81:1-6

19. Nübling M, Stößel U, Hasselhorn H-M et al (2004) Mitarbeiterbefragungen zu psychosozialen Belastungen in Betrieben - Das Befragungsinstrument COPSOQ. In: Hofmann F, Reschauer G, Stößel U (Hrsg) Arbeitsmedizin im Gesundheitsdienst. Tagungsband 17 des Freiburger Symposiums Arbeitsmedizin im Gesundheitsdienst. Edition FFAS, Freiburg, S227-242

20. Nübling $M$, Stößel $U$, Hasselhorn $H M$ et a (2005) Methoden zur Erfassung psychischer Belastungen. Bundesanstalt für Arbeitsschutz und Arbeitsmedizin, Dortmund, Berlin, Dresden

21. Pejtersen JH, Kristensen TS, Borg V et al (2010) The second version of the Copenhagen psychosocial questionnaire. Scand J Public Health 38:8-24

22. Ramos R, Jenny G, Bauer G (2016) Age-related effects of job characteristics on burnout and work engagement. Occup Med 66:230-237

23. Rose U, Müller G, Freude G et al (2019) Arbeitsbedingungen und psychische Gesundheit bei sozialversicherungspflichtig beschäftigten Ärzten: Ein bundesweiter Vergleich mit einer repräsentativen Beschäftigtenstichprobe. Gesundheitswesen 51:382-390

24. Rose U, Schiel S, Schröder H et al (2017) The study on mental health at work: design and sampling. Scand J Public Health 45:584-594

25. Schaufeli WB, Enzmann D (1998) The burnout companion to study and practice. A critical analysis. Taylor \& Francis, London

26. Schröder H, Köhler T, Knerr P et al (2015) Einfluss psychischer Belastungen am Arbeitsplatz auf das Neuroenhancement - empirische Untersu- chungen an Erwerbstätigen. Bundesanstalt für Arbeitsschutz und Arbeitsmedizin, Dortmund, Berlin, Dresden

27. Schröder H, Schiel S, Schulz S et al (2015) Mentale Gesundheit bei der Arbeit (S-MGA): Methodenbericht zur Repräsentativerhebung an Erwerbstätigen in Deutschland. Bundesanstalt für Arbeitsschutz und Arbeitsmedizin, Dortmund, Berlin, Dresden

28. Seidler A, Thinschmidt M, Deckert S et al (2014) The role of psychosocial working conditions on burnout and its core component emotional exhaustion - a systematic review. J Occup Med Toxicol 9:1-10

29. Stab N, Jahn S, Schulz-Dadaczynski A (2016) Psychische Gesundheit in der Arbeitswelt Arbeitsintensität. Bundesanstalt für Arbeitsschutz und Arbeitsmedizin, Dortmund, Berlin, Dresden

30. Wöhrmann AM, Gerstenberg $S$, Hünefeld $L$ et al (2016) Arbeitszeitreport Deutschland 2016. Bundesanstalt für Arbeitsschutz und Arbeitsmedizin Dortmund, Berlin, Dresden 